\title{
A new critical plane-energy model for multiaxial fatigue life prediction of turbine disc alloys
}

\author{
Shen $\mathrm{Xu}^{\mathrm{a}}$, Shun-Peng Zhu ${ }^{\mathrm{a}, \mathrm{b}, *}$, Yong-Zhen $\mathrm{Hao}^{\mathrm{a}}$, Ding Liao ${ }^{\mathrm{a}}$, Guian Qian ${ }^{\mathrm{c}}$ \\ a School of Mechanical and Electrical Engineering, University of Electronic Science and Technology of China, Chengdu 611731, China \\ ${ }^{\mathrm{b}}$ Center for System Reliability \& Safety, University of Electronic Science and Technology of China, Chengdu 611731, China \\ ${ }^{\mathrm{c}}$ State Key Laboratory of Nonlinear Mechanics (LNM), Institute of Mechanics, Chinese Academy of Sciences, Beijing 100190, China
}

\section{A R T I C L E I N F O}

\section{Keywords:}

Multiaxial fatigue

Virtual strain energy

Critical plane

Mean stress

Life prediction

\begin{abstract}
A B S T R A C T
For engine components under complex loadings, multiaxial fatigue life prediction is critical for ensuring their structural integrity and reliability. Combining the critical plane method with the virtual strain energy concept, a new multiaxial fatigue damage parameter is proposed to characterize the influence of both shear/normal mean stress and non-proportional hardening on fatigue life. Particularly, no extra material constants are needed for model application. Experimental data of TC4 and GH4169 alloys under various loading paths are utilized to evaluate and validate the proposed damage parameter as well as four other models. Results show that the proposed damage parameter yields a higher accuracy on multiaxial fatigue life prediction than others.
\end{abstract}

\section{Introduction}

The integrity of aero engine components in service are mainly threatened by multiaxial fatigue failure due to their various multiaxial loading paths [1-6]. Accordingly, increasing attentions are being paid on developing valid approaches for multiaxial fatigue analysis [7-14]. Among them, most multiaxial fatigue models were presented based on simple combinations of stresses or strains $[15,16]$. Though a scalar parameter might provide a reasonable life prediction, it cannot explain well the experimental phenomenon that cracks initiate and grow on a specific plane rather than a random one [17]. Based on this, the critical plane-based methods have been introduced for practice, in which the critical plane is normally defined differently for different multiaxial fatigue damage parameters.

In particular, various multiaxial fatigue models have been put forward through combining the critical plane-based method with stress/strain-based parameters [17] as well as strain energy-based damage parameters, which combine the effects of loading histories and both states of stress and strain. Among them, Garud [18] applied the plastic strain energy density for multiaxial fatigue analyses. However, this plastic work is really small in a high cycle fatigue (HCF) regime and is difficult to measure or calculate for model application with sufficient accuracy. According to this, Liu [19] developed two damage parameters on the planes with the maximum amount of axial/shear work component to consider both tensile/shear failure modes, which contains elastic and plastic parts to overcome the limitations of Garud's model, but the need of judging failure mode causes the inconvenience for model application. Then, Chu [20] introduced a similar parameter to combine shear and normal works and replaced the stress range with the maximum stress to account for the effect of mean stress, however, ignored the mean stress effect in the case of non-proportional loading with

\footnotetext{
* Corresponding author at: School of Mechanical and Electrical Engineering, University of Electronic Science and Technology of China, Chengdu 611731, China.

E-mail address: zspeng2007@uestc.edu.cn (S.-P. Zhu).
} 
zero strain amplitude. Thus, a stable and effective multiaxial fatigue criterion is still lacking for engineering practice.

In this regard, through considering the virtual shear strain energy and the normal stress term, this work aims to present a new critical plane-strain energy damage parameter for fatigue lifing of engine components. For the rest of this paper, a short review on several commonly-used multiaxial fatigue damage parameters is briefly presented in Section 2. Section 3 defines the maximum virtual shear strain-energy plane as the critical plane and develops a new multiaxial fatigue damage parameter. In Section 4, experimental data of TC4 and GH4169 alloys under different loading paths are utilized for model validation. Section 5 draws the conclusions.

\section{Existing damage parameters for multiaxial fatigue analysis}

As aforementioned, various damage parameters have been presented for multiaxial fatigue analysis, including stress-based, strainbased and strain energy-based ones. Particularly, several commonly-used ones are introduced as follows.

i) McDiarmid's criterion

McDiarmid [21] analyzed the same fatigue data used by Findley [22], but came to a slightly different model. He replaced Findley's material parameter $k$ with the quantity $\frac{t_{A, B}}{2 \sigma_{u t s}}$ to avoid the inconvenience of defining $k$ and developed a stress-based criterion as.

$$
\frac{\Delta \tau_{\max }}{2 t_{A, B}}+\frac{\sigma_{n, \max }}{2 \sigma_{u t s}}=1
$$

With regard to fatigue life prediction, Eq. (1) can be rewritten according to the Basquin's equation by

$$
\frac{\Delta \tau_{\max }}{2}+\left(\frac{t_{A, B}}{2 \sigma_{u t s}}\right) \sigma_{n, \max }=\tau_{f}^{\prime}\left(2 N_{f}\right)^{b_{0}}
$$

where $\Delta \tau_{\max }$ is the maximum shear stress range, $\sigma_{n, \max }$ is the maximum normal stress on the same plane, $t_{A, B}$ is the fatigue strength for Case A (acting on the free surface) or Case B (growing into the depth) cracking and $\sigma_{\text {uts }}$ is the ultimate tensile strength. Eq. (2) characterizes both A and B case cracking modes. Case A cracking propagates along the free surface, and Case B cracking results in cracks that penetrate into the material. In the McDiarmid's damage parameter, the critical plane is defined by the plane with maximum shear stress. However, most stress-based models, including models of Findley [22] and Dang Van [23], can only provide acceptable life prediction results in the HCF regime due to the small plastic deformation.

\section{ii) WB and FS criteria}

On the basis of the research of Brown and Miller [24], Wang and Brown [25] introduced a maximum shear strain plane and modified the normal strain on the same plane. Furthermore, they considered the mean stress effect on fatigue life and put forward a strain-based criterion, namely the WB damage parameter, which can be expressed by

$$
\begin{gathered}
\frac{\Delta \gamma_{\max }}{2}+S \Delta \varepsilon_{n}=A^{\prime} \frac{\sigma_{f}^{\prime}-2 \sigma_{n, \text { mean }}}{E}\left(2 N_{f}\right)^{b}+B^{\prime} \varepsilon_{f}^{\prime}\left(2 N_{f}\right)^{c} \\
\text { where } A^{\prime}=1+v_{e}+\left(1-v_{e}\right) S \\
B^{\prime}=1+v_{p}+\left(1-v_{p}\right) S
\end{gathered}
$$

where $\Delta \gamma_{\max }$ is the maximum shear strain range, $\Delta \varepsilon_{n}$ is the normal strain range on the plane experiencing the maximum shear strain, $\sigma_{n, \text { mean }}$ is the normal mean stress on the plane, $E$ is the elastic modulus, $v_{e}$ and $v_{p}$ are the elastic and plastic Poisson's ratios, and $S$ is an additional material constant that accounts for the influence of normal strain on fatigue cracking.

Based on Eq. (3), Fatemi and Socie [26] replaced normal strain term with the normal stress, which leads to the FS damage parameter as

$$
\frac{\Delta \gamma_{\max }}{2}\left(1+k \frac{\sigma_{n, \max }}{\sigma_{y}}\right)=\frac{\tau_{f}^{\prime}}{G}\left(2 N_{f}\right)^{b_{0}}+\gamma_{f}^{\prime}\left(2 N_{f}\right)^{c_{0}}
$$

where $\sigma_{y}$ is the cyclic yield strength [27], $k$ is an additional material constant defined to reflect the effect of normal stress on crack growth. Though both the WB and FS models evaluate fatigue life for various ductile materials with an acceptable prediction ability, it is inconvenient to determine the value of $S$ or $k$ by fitting axial and torsion data, particularly under limited testing data conditions. In addition, its additional material constants calculated by different ways usually lead to different life predictions.

\section{iii) SWT and MSWT models}

Smith, Watson and Topper [28] considered the effect of both the principal strain range and the maximum stress, then introduced an alternate damage model, namely the SWT model. 


$$
\sigma_{n, \max } \frac{\Delta \varepsilon_{1}}{2}=\frac{\sigma_{f}^{\prime 2}}{E}\left(2 N_{f}\right)^{2 b}+\sigma_{f}^{\prime} \varepsilon_{f}^{\prime}\left(2 N_{f}\right)^{b+c}
$$

where $\Delta \varepsilon_{1}$ is the principal strain range. The critical plane of SWT model corresponds to the principal strain. The stress term makes it suitable to describe the mean stress during multiaxial load paths and non-proportional hardening effects. However, note that the SWT model only considers the normal strain energy in fatigue life prediction, but ignores the effect of the shear components. Thus, the SWT criterion is usually used for materials under uniaxial loading situations in which fatigue fracture primarily on account of the Mode I tensile cracking [28].

Recently, several energy-based damage parameters have been developed based on the SWT model, such as Chu [20], Glinka et al. [29], etc. Particularly, for applying to the general cracking mode, Jiang and Sehitoglu [30] extended the SWT criterion by increasing a shear strain energy term, and presented the modified SWT (MSWT) damage parameter as:

$$
2 a \Delta \varepsilon\left\langle\sigma_{\max }\right\rangle+\frac{1-a}{2} \Delta \tau \Delta \gamma=f\left(N_{f}\right)
$$

where $\Delta \tau$ and $\Delta \gamma$ are the shear stress/strain range on the critical plane, respectively. The symbol $\langle$ is the MacCauley bracket, defined as $\langle x\rangle=0.5(\mathrm{x}+|x|)$, which ensures the positive fatigue damage. $a$ is an additional material constant ranging from zero to one. The critical plane of MSWT model is defined as the maximum damage parameter plane.

Note from [31] that the MSWT model can predict different cracking behavior with different value of $a$ in Eq. (6). When $0 \leq a \leq 0.37$, shear crack behavior is predicted, and $a \geq 0.5$ for tensile crack behavior, and $0.37<a<0.5$ for mixed cracking behavior.

Then, Ma et al. [32] estimated the fatigue life based on the uniaxial fatigue data by

$$
f\left(N_{f}\right)=4 a \frac{\sigma_{f}^{\prime 2}}{E}\left(2 N_{f}\right)^{2 b}+4 a \sigma_{f}^{\prime} \varepsilon_{f}^{\prime}\left(2 N_{f}\right)^{b+c}
$$

It's worth noting that when $a=1$, the MSWT criterion reduces to the original SWT criterion.

\section{Proposed critical plane-energy damage parameter}

In general, stress-based parameters are mainly applied for HCF life prediction, and contrariwise, strain-based parameters for lowcycle fatigue (LCF) life prediction [33-42]. Therefore, energy-based damage parameters are becoming more popular in recent researches due to its good ability for both HCF and LCF life prediction.

For considering both stress and strain during loadings, Liu [19] put forward a concept of virtual strain energy (VSE) based on the hysteresis loop energy and divided the VSE on a plane into elastic/plastic components. For the shear work, the virtual shear strain energy (VSSE) can be calculated by.

$$
\begin{aligned}
& E_{S}=E_{S}^{e}+E_{S}^{p} \cong \Delta \tau \Delta \gamma \\
& E_{S}^{p} \cong \Delta \tau \Delta \gamma^{p} \\
& E_{S}^{e}=\Delta \tau \Delta \gamma^{e}
\end{aligned}
$$

where $E_{S}$ represents the VSSE on the same plane. The superscript $e$ and $p$ denote the elastic/plastic work, respectively.

However, the VSSE is unable to correlate test data from both tension and torsion. For TC4 alloy, Fig. 1 depicts a correlation between the maximum VSSE and the tested life under different loading conditions. Note that the maximum VSSE calculated by the

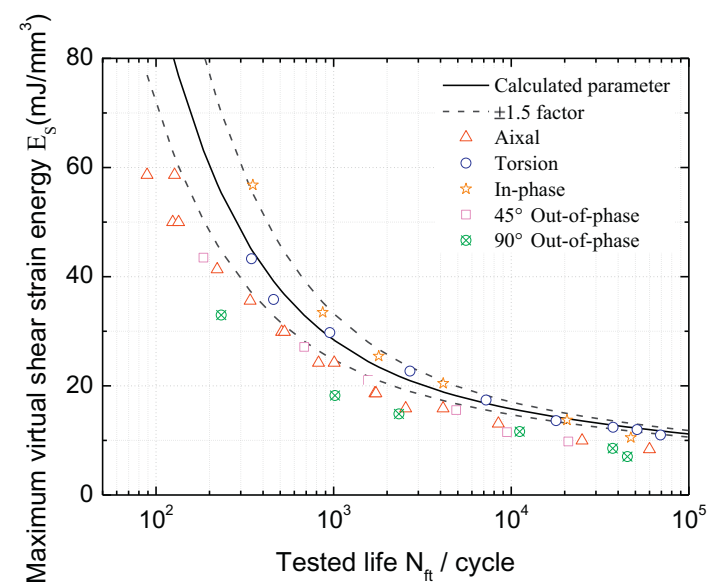

Fig. 1. Maximum virtual shear strain energy $E_{s}$ vs. tested life $N_{f t}$ for TC4. 
tested life $N_{f t}$ under torsion and in-phase loadings are close to the curve, and smaller under other loading paths, which indicates that under these loading conditions, the normal work also has shown a promoting effect on fatigue damage accumulation, and it becomes more obvious with the increasing of test life $N_{f t}$. Consequently, the undervalued fatigue damage parameter tends to overestimate fatigue life of TC4 alloys since it ignores the influence of normal stress or strain on crack growth.

Note that the FS criterion considers the effect of normal work by adding a normal stress term and provides acceptable life predictions for various materials. Since the maximum normal stress $\sigma_{n, \max }$ on the critical plane can not only take account of the influence of mean stress, but also increases when materials show non-proportional hardening [12]. However, it ignores the effect of shear stress on fatigue damage [20]. Thus, through replacing the shear strain term with virtual shear strain energy, a critical plane fatigue parameter based on the FS criterion can be derived as.

$$
E_{\text {Smax }}\left(1+k \frac{\sigma_{n, \max }}{\sigma_{y}}\right)=f\left(N_{f}\right)
$$

where $E_{S \max }$ is the maximum VSSE on the critical plane, $\sigma_{y}$ is the material yield strength. Moreover, the critical plane of this analysis is defined by the maximum virtual shear strain-energy plane which considers both stress and strain components.

Noticed from [43], the coefficient $k$ can be estimated by $k \approx \sigma_{y} / \sigma_{f}^{\prime}$ under limited data conditions, which simplifies the calculation of the additional material constant $k$. Thus, Eq. (11) can be rewritten as

$$
E_{\text {Smax }}\left(1+\frac{\sigma_{n, \max }}{\sigma_{f}^{\prime}}\right)=f\left(N_{f}\right)
$$

Since the damage parameter in Eq. (12) is built on the shear work, it is reasonable to utilize the shear strain-life properties on the critical plane, which can be given by [12].

$$
\begin{aligned}
& \frac{\Delta \gamma}{2}=\frac{\tau_{f}^{\prime}}{G}\left(2 N_{f}\right)^{b_{0}}+\gamma_{f}^{\prime}\left(2 N_{f}\right)^{c_{0}} \\
& \frac{\Delta \tau}{2}=\tau_{f}^{\prime}\left(2 N_{f}\right)^{b_{0}} \\
& \sigma_{n, \max }=0
\end{aligned}
$$

Through substituting Eqs. (13)-(15) into Eq. (12), a critical plane-energy model for multiaxial fatigue analyses can be derived as.

$$
E_{\text {Smax }}\left(1+\frac{\sigma_{n, \max }}{\sigma_{f}^{\prime}}\right)=4 \frac{\tau_{f}^{\prime 2}}{G}\left(2 N_{f}\right)^{2 b_{0}}+4 \tau_{f}^{\prime} \gamma_{f}^{\prime}\left(2 N_{f}\right)^{b_{0}+c_{0}}
$$

Note from Eq. (16) that the proposed damage parameter mainly considers the shear work component and includes mean stress correction to explain crack surface sliding and propagating [44-46]. It's worth mentioning that for shear failure mode, which is fractured primarily by Mode II cracking, shear strain energy is the primary driving force of crack initiation and early growth by overcoming sliding friction, and the normal stress assists the crack opening process $[47,48]$. In particular, fatigue fracture mechanism of the proposed damage parameter is depicted in Fig. 2.

It's worth noting that no additional material constants are needed when applying Eq. (16) for multiaxial fatigue life prediction.

\section{Experimental validation and discussion}

\subsection{Material properties}

For evaluation and validation of the proposed energy-based damage parameter, experimental data of TC4 and GH4169 alloys are utilized [49,50]. For these two alloys, the solid specimens were forged for monotonic/axial fatigue tests according to Chinese standard GB/T 15248-2008, and the tubular specimens were manufactured for pure torsional and multiaxial fatigue tests according to the ASTM E2207 standard. More details on the design and fabrication work of TC4 and GH4169 samples can be referred to [49,50]. Material properties of the two alloys are respectively summarized in Tables 1 and 2.

It is worth noting that all experiments were carried out under various strain-controlled load paths, including axial, torsional, inphase and $45^{\circ} / 90^{\circ}$ out-of-phase loading conditions. Sine waveforms with testing frequency $(0.1-1.0 \mathrm{~Hz})$ were conducted for the case

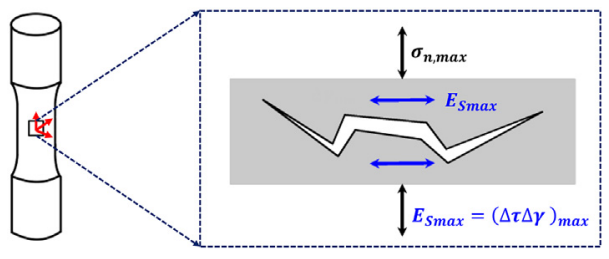

Fig. 2. Crack mechanism of the proposed model. 
Table 1

Fatigue properties of TC4 alloy.

\begin{tabular}{lllllll}
\hline Monotonic & $E(\mathrm{GPa}) 108.4$ & $\mathrm{G}(\mathrm{GPa}) 43.2$ & $\sigma_{y}(\mathrm{MPa}) 942.5$ & $v_{e} 0.25$ & $K(\mathrm{MPa}) 1054$ & $n 0.0195$ \\
\hline Uniaxial & $\sigma_{f}^{\prime}(\mathrm{MPa}) 942.5$ & $b-0.049$ & $\varepsilon_{f^{\prime}} 0.579$ & $c-0.679$ & $K^{\prime}(\mathrm{MPa}) 1031$ & $n^{\prime} 0.0478$ \\
Torsional & $\tau_{f}^{\prime}(\mathrm{MPa}) 716.9$ & $b_{0}-0.06$ & $\gamma_{f}^{\prime} 2.24$ & $c_{0}-0.8$ & $K_{0}^{\prime}(\mathrm{MPa}) 446.7$ & $n_{0}^{\prime} 0.016$ \\
\hline
\end{tabular}

Table 2

Fatigue properties of GH4169 alloy.

\begin{tabular}{|c|c|c|c|c|c|c|}
\hline Monotonic & $E(\mathrm{GPa}) 198.5$ & G(GPa) 67 & $\sigma_{y}(\mathrm{MPa}) 1083.1$ & $v_{e} 0.48$ & K(MPa) 1579.7 & $n 0.06$ \\
\hline Uniaxial & $\sigma_{f}^{\prime}(\mathrm{MPa}) 1815.5$ & $b-0.06$ & $\varepsilon_{f}^{\prime} 0.45$ & $c-0.63$ & $K^{\prime}(\mathrm{MPa}) 1892.3$ & $n^{\prime} 0.078$ \\
\hline Torsional & $\tau_{f}^{\prime}(\mathrm{MPa}) 1091.6$ & $b_{0}-0.07$ & $\gamma_{f}^{\prime} 4.46$ & $c_{0}-0.77$ & $K_{0}^{\prime}(\mathrm{MPa}) 1047.1$ & $n_{0}^{\prime} 0.099$ \\
\hline
\end{tabular}

of symmetric/asymmetric loading conditions for TC4 testing. For GH4169 alloy, fatigue tests were controlled under fully reversed sine wave in strain-controlled mode with frequency of $0.5-1.0 \mathrm{~Hz}$.

\subsection{Model comparison and discussions}

During multiaxial fatigue analysis, Socie and Marquis [12] pointed out that valid models should include the parameters for cyclic plasticity, state of stress, non-proportional loading and mean stress, which influence the damage mechanisms. In this section, four other critical plane-based damage parameters are introduced for model comparison, including WB, FS, SWT, and MSWT damage parameters.

For the two alloys, model comparisons were performed as shown in Figs. 3-5. Moreover, the model prediction error is defined and calculated by [51-54]:

$$
P_{\text {error }}=\log _{10}\left(N_{f p}\right)-\log _{10}\left(N_{f t}\right)
$$

where $N_{f p}$ and $N_{f t}$ are the predicted and tested life, respectively.

As shown in Figs. 3-4, the aforementioned models all provide reasonable fatigue life prediction results for TC4 and GH4169 during symmetric load paths, except the SWT model. That's because the SWT model only considers the growth of microcracks in Mode I, which neglects the effect of shear work on damage mechanism. Thus, the SWT damage parameter will be extremely small when materials fail primarily in Mode II cracking, which leads to the overestimation of life prediction under pure torsion and nonproportional loadings. It is worth mentioning that the modified SWT model overcomes this issue by adding a shear strain-energy term. Moreover, note from Figs. 3(a) and 4(a) that the WB model shows a good life prediction ability for TC4 alloy, but provides overconservative fatigue life predictions for GH4169 alloy. However, Figs. 3(b) and 4(b) present the performance of the FS model is contrary to that of the WB model. As for the MSWT model, Fig. 3(d) shows its more accurate life prediction results for TC4 than the SWT model. As shown in Fig. 4(d), it provides conservative life predictions of GH4169 just like the WB model, especially during inphase and $45^{\circ} / 90^{\circ}$ out-of-phase loading paths. This stems from the fact that the effect of normal strain is overestimated on fatigue damage for GH4169, which leads to conservative life prediction results. The proposed model overcomes shortcomings of the abovementioned three models, while as presented in Figs. 3(e) and 4(e), the proposed damage parameter predicts fatigue lives almost all within a life factor of 3 for both TC4 and GH4169 alloys, although the life prediction of GH4169 is slightly conservative comparing with that of FS damage parameter.

Furthermore, whether or not to consider the mean stress effect is also an important factor to validate the damage parameter for life prediction. Fig. 5 shows the result of comparing multiaxial fatigue life predictions among these five models for TC4 under asymmetrical loading paths. Note that life predictions with the proposed damage parameter are all within or near the life scatter band of \pm 3 , with only exception of two data points, as well as the MSWT model. For the WB model, it also provides acceptable life predictions on the whole, but has two extremely overpredicted data points under $90^{\circ}$ out-of-phase loading.

As shown in Fig. 5(b), note that the MSWT and proposed model yield better predictions than others as noticed by the lower mean/ scatter of $P_{\text {error }}$. Since both the MSWT and proposed damage parameters include a shear strain energy component to account for the shear mean stress effect on fatigue damage, while the normal strain energy component $\Delta \varepsilon\left\langle\sigma_{\max }\right\rangle$ in the MSWT parameter and $\sigma_{n, \max }$ in the proposed damage parameter make them suitable for describing normal mean stress and non-proportional hardening effects. However, the three other damage parameters cannot deal with the effect of shear mean stress under multiaxial loadings. In addition, with no additional material coefficients, the proposed damage parameter is more convenient for practice application than the WB, FS and MSWT parameters, especially for multiaxial fatigue analysis under shear failure mode.

\section{Conclusion}

This paper defined a critical plane with the maximum VSSE and put forward a new damage parameter for multiaxial fatigue analysis considering the shear and normal mean stress on the critical plane. The main conclusions are summarized as below: 
(a)
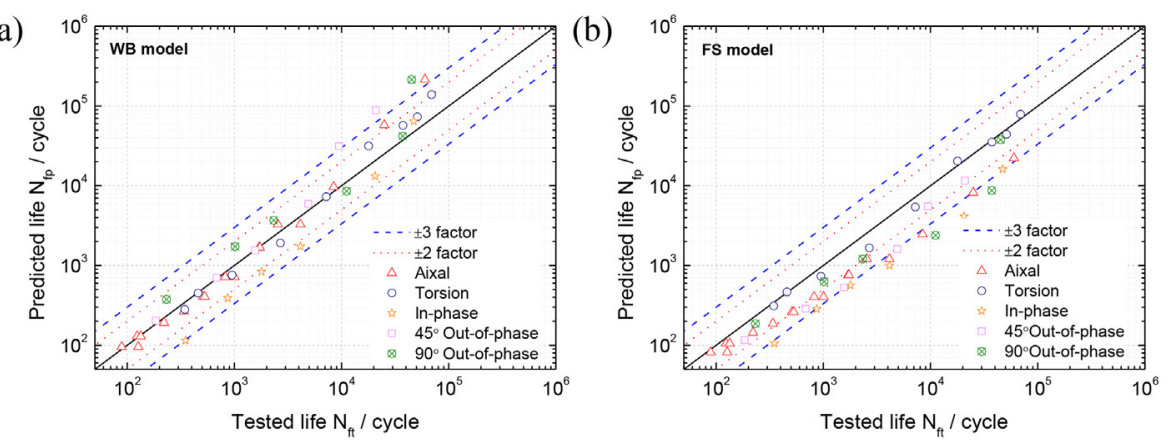

(c)

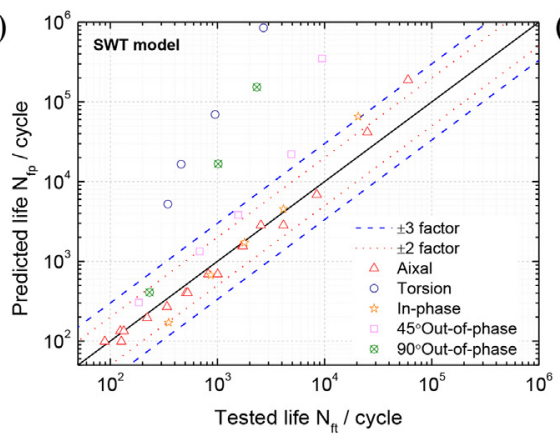

(d)

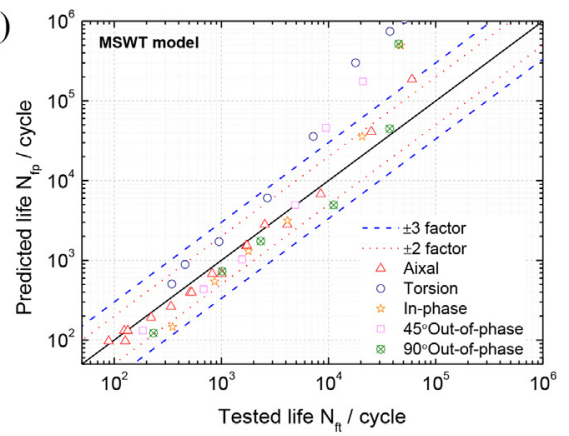

(e)

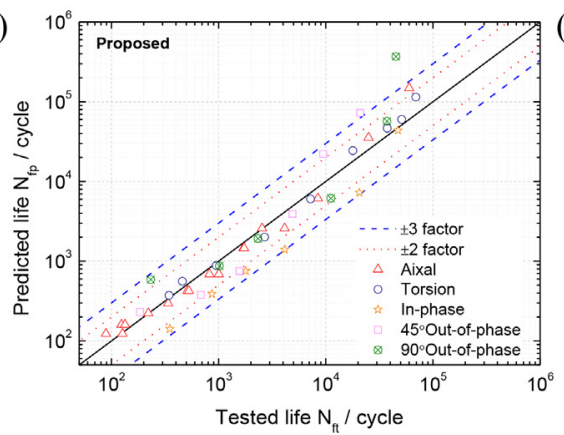

(f)

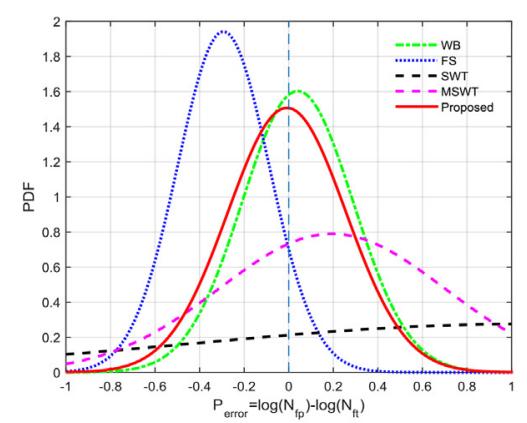

Fig. 3. Life prediction results for TC4 by models of (a) WB, (b) FS, (c) SWT, (d) MSWT and (e) the proposed model under symmetric loadings and (f) model prediction errors.

(1) A new and simple multiaxial fatigue model without requirement of additional material constants is proposed by combining critical plane and strain energy concepts.

(2) For TC4 and GH4169 alloys under different loading paths, the SWT model provides accurate life prediction results under uniaxial loadings, but poor performance under multiaxial loadings. However, the MSWT model overcomes this issue and performs really better for multiaxial fatigue life prediction of TC4 alloy.

(3) The proposed model gives better correlations with experimental results of TC4 and GH4169 alloys, which indicated that including both shear strain and shear stress in the main control parameter yields more accurate life prediction.

\section{Nomenclature}

$\gamma_{\mathrm{a}} \quad$ Maximum shear strain amplitude on the critical plane

$\Delta \tau_{\max } \quad$ Maximum shear strain rang on the critical plane

$\sigma_{n, \max } \quad$ Maximum normal stress on the critical plane

$t_{A, B} \quad$ Fatigue strength for Case A or Case B cracking

$\sigma_{u t s} \quad$ Ultimate tensile strength

$\Delta \varepsilon_{n} \quad$ Normal strain range on the critical plane

$S \quad$ Wang-Brown parameter

$\sigma_{n, \text { mean }}$ Normal mean stress on the critical plane

$\sigma_{y} \quad$ Cyclic yield stress

$k \quad$ Fatemi-Socie parameter 
(a)

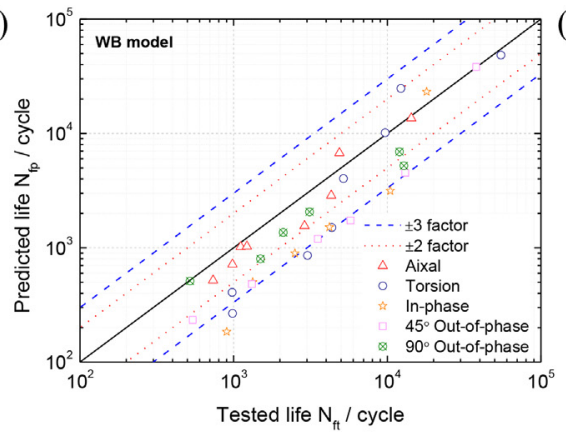

(c)

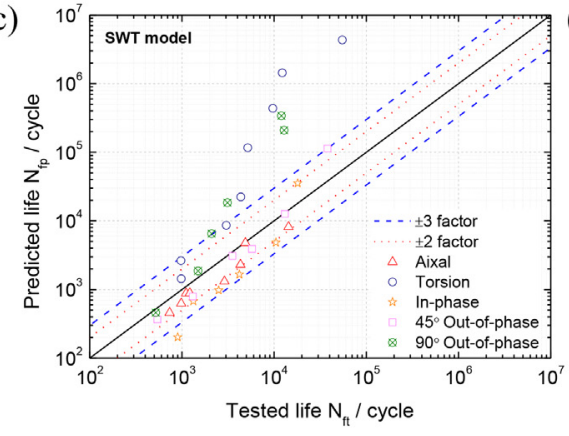

(e)

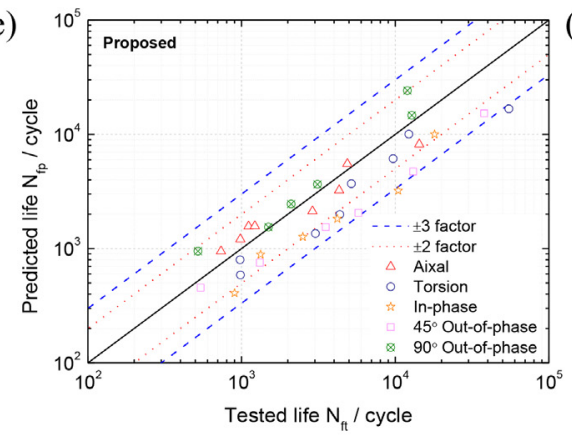

(b)

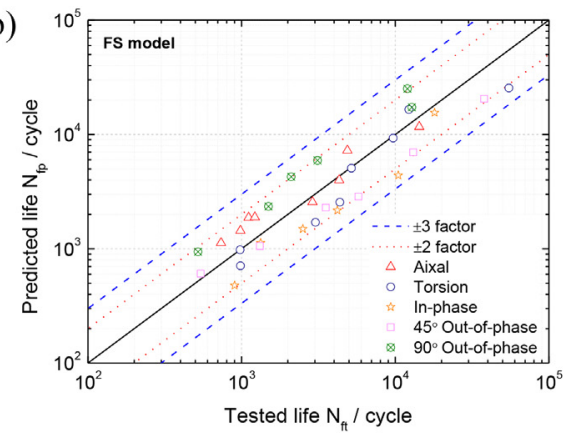

(d)

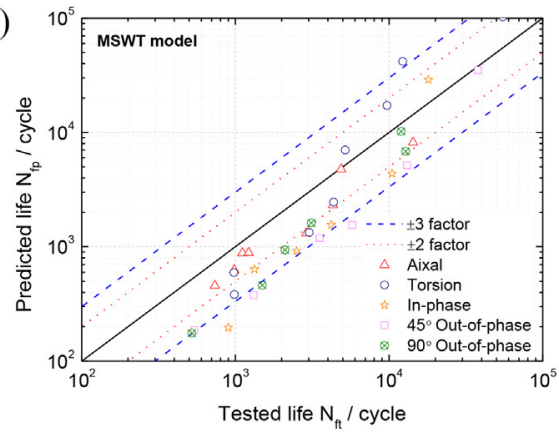

(f)

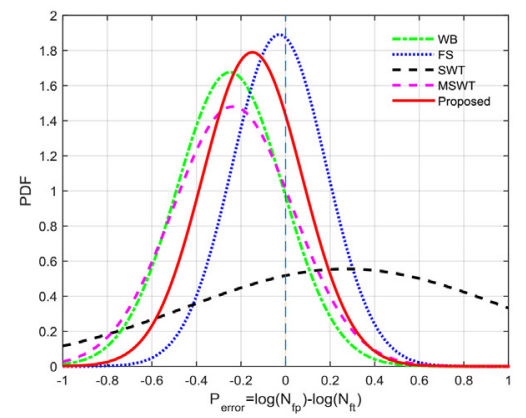

Fig. 4. Life prediction results for GH4169 by models of (a) WB, (b) FS, (c) SWT, (d) MSWT and (e) the proposed model under symmetric loadings and (f) model prediction errors.

(a)

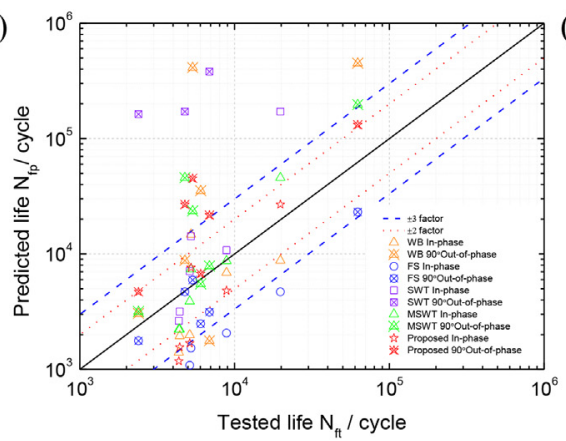

(b)

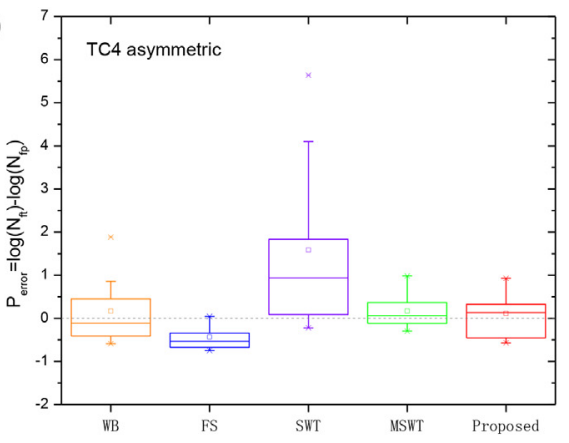

Fig. 5. (a) Model predictions and (b) box plot of $P_{\text {error }}$ for TC4 under asymmetric loadings

$\Delta \varepsilon_{1} \quad$ Principal strain range

$\tau_{f}^{\prime} \quad$ Shear fatigue strength coefficient

$\gamma_{f}^{\prime} \quad$ Shear fatigue ductility coefficient

$N_{f} \quad$ Number of cycles to failure

a Modified SWT parameter

$G \quad$ Shear modulus 
$b_{0} \quad$ Shear fatigue strength exponent

$c_{0} \quad$ Shear fatigue ductility exponent

$v_{e} \quad$ Elastic Poisson's ratio

$v_{p} \quad$ Plastic Poisson's ratio

$\sigma_{f}^{\prime} \quad$ Fatigue strength coefficient

$\varepsilon_{f}^{\prime} \quad$ Fatigue ductility coefficient

$E \quad$ Young modulus

b Fatigue strength exponent

c Fatigue ductility exponent

\section{Acknowledgement}

The financial support of the National Natural Science Foundation of China (No. 11672070 and 11302044), Fundamental Research Funds for the Central Universities (No. ZYGX2016J208), China Postdoctoral Science Foundation Funded Project (No. 2015M582549 and 2017T100697) are acknowledged.

\section{References}

[1] W. Maktouf, K. Sai, An investigation of premature fatigue failures of gas turbine blade, Eng. Fail. Anal. 47 (2015) 89-101.

[2] D. Hu, R. Wang, Combined fatigue experiments on full scale turbine components, Aircr. Eng. Aerosp. Technol. 85 (1) (2013) 4-9.

[3] S.P. Zhu, H.Z. Huang, W. Peng, H.K. Wang, S. Mahadevan, Probabilistic physics of failure-based framework for fatigue life prediction of aircraft gas turbine discs under uncertainty, Reliab. Eng. Syst. Saf. 146 (2016) 1-12.

[4] S.P. Zhu, Q. Liu, Q. Lei, Q.Y. Wang, Probabilistic fatigue life prediction and reliability assessment of a high pressure turbine disc considering load variations, Int. J. Damage Mech. (2018) in press https://doi.org/10.1177/1056789517737132.

[5] S.P. Zhu, Q. Liu, J. Zhou, Z.Y. Yu, Fatigue reliability assessment of turbine discs under multi-source uncertainties, Fatigue Fract. Eng. 41 (2018) $1291-1305$.

[6] R.Z. Wang, X.C. Zhang, S.T. Tu, S.P. Zhu, C.C. Zhang, A modified strain energy density exhaustion model for creep-fatigue life prediction, Int. J. Fatigue 90 (2016) $12-22$.

[7] J.A.F.O. Correia, et al., A generalization of the fatigue Kohout-Věchet model for several fatigue damage parameters, Eng. Fract. Mech. 185 (2017) 284-300.

[8] R. Wang, H. Liu, D. Hu, et al., Evaluation of notch size effect on LCF life of TA19 specimens based on the stress gradient modified critical distance method, Fatigue Fract. Eng. Mater. Struct. (2018) in press https://doi.org/10.1111/ffe.12821.

[9] Z.R. Wu, X. Li, L. Fang, et al., Multiaxial fatigue life prediction based on nonlinear continuum damage mechanics and critical plane method, J. Mater. Eng. Perform. (2018) in press https://doi.org/10.1007/s11665-017-3045-4.

[10] S.P. Zhu, P. Yue, Z.Y. Yu, Q. Wang, A combined high and low cycle fatigue model for life prediction of turbine blades, Materials 10 (7) (2017) 698.

[11] S.P. Zhu, Y.J. Yang, H.Z. Huang, Z. Lv, H.K. Wang, A unified criterion for fatigue-creep life prediction of high temperature components, Proc. Inst. Mech. Eng. G 231 (4) (2017) 677-688.

[12] D.F. Socie, G.B. Marquis, Multiaxial Fatigue, SAE Inc., 2000.

[13] S.P. Zhu, Y. Liu, Q. Liu, Z.Y. Yu, Strain energy gradient-based LCF life prediction of turbine discs using critical distance concept, Int. J. Fatigue 113 (2018) 33-42.

[14] S.P. Zhu, Z.Y. Yu, J. Correia, A. De Jesus, F. Berto, Evaluation and comparison of critical plane criteria for multiaxial fatigue analysis of ductile and brittle materials, Int. J. Fatigue 112 (2018) 279-288.

[15] Y.S. Garud, A new approach to the evaluation of fatigue under multiaxial loadings, J. Manuf. Sci. 103 (2) (1981) $118-125$.

[16] F. Ellyin, K. Golos, Multiaxial fatigue damage criterion, J. Mater. Sci. Technol. 110 (1) (1988) 63-68.

[17] C. Lu, J. Melendez, J.M. Martínez-Esnaola, Fatigue damage prediction in multiaxial loading using a new energy-based parameter, Int. J. Fatigue 104 (2017) 99-111.

[18] Y.S. Garud, A new approach to the evaluation of fatigue under multiaxial loadings, J. Eng. Mater. Technol. 103 (2) (1981) 118-125. Transactions of the ASME.

[19] K.C. Liu, A method based on virtual strain-energy parameters for multiaxial fatigue life prediction, Advances in multiaxial fatigue, American Society for Testing and Materials STP 1191, Philadelphia, 1993, pp. 67-84.

[20] C.C. Chu, Fatigue damage calculation using the critical plane approach, J. Eng. Mater. Technol. 117 (1) (1995) $41-49$.

[21] D.L. Mcdiarmid, A shear stress based critical-plane criterion of multiaxial fatigue failure for design and life prediction, Fatigue Fract. Eng. M. 17 (12) (1994) $1475-1484$.

[22] W.N. Findley, Modified theories of fatigue failure under combined stress, Proc. Soc. Exp. Stress Anal. 14 (1) (1956) 35-46.

[23] K.D. Van, G. Cailletaud, J.F. Flavenot, et al., "Criterion for High-cycle Fatigue Failure under Multiaxial Loading," ICBMFF2, (1986).

[24] M.W. Brown, K.J. Miller, A theory for fatigue failure under multiaxial stress-strain conditions, Arch. Proc. Inst. Mech. Eng. 187 (1973) 745-755, 2006.

[25] C.H. Wang, M.W. Brown, Multiaxial random load fatigue: life prediction techniques and experiments, Multiaxial Fatigue Des. 21 (1996) 513-527.

[26] A. Fatemi, D.F. Socie, A critical plane approach to multiaxial fatigue damage including out-of-phase loading, Fatigue Fract. Eng. Mater. Struct. 11 (3) (1988) $149-165$.

[27] Z.Y. Yu, S.P. Zhu, Q. Liu, et al., Multiaxial fatigue damage parameter and life prediction without any additional material constants, Materials 10 (8) (2017) 923.

[28] K.N. Smith, P. Watson, T.H. Topper, A stress-strain function for the fatigue of metals, J. Mater. 5 (1970) 767-778.

[29] G. Glinka, G. Wang, A. Plumtree, Mean stress effects in multiaxial fatigue, Fatigue Fract. Eng. Mater. Struct. 18 (7) (1995) $755-764$.

[30] Y. Jiang, O. Hertel, M. Vormwald, An experimental evaluation of three critical plane multiaxial fatigue criteria, Int. J. Fatigue 29 (8) (2007) 1490-1502.

[31] T. Zhao, Y. Jiang, Fatigue of 7075-T651 aluminum alloy, Int. J. Fatigue 30 (2008) 834-849.

[32] S. Ma, B. Markert, H. Yuan, Multiaxial fatigue life assessment of sintered porous iron under proportional and non-proportional loadings, Int. J. Fatigue 97 (2017) $214-226$.

[33] S.P. Zhu, Q. Liu, W. Peng, X.C. Zhang, Computational-experimental approaches for fatigue reliability assessment of turbine bladed disks, Int. J. Mech. Sci. 142143 (2018) 502-517.

[34] R.Z. Wang, S.P. Zhu, J. Wang, X.C. Zhang, S.T. Tu, C.C. Zhang, High temperature fatigue and creep-fatigue behaviors in a Ni-based superalloy: damage mechanisms and life assessment, Int. J. Fatigue (2018) in press https://doi.org/10.1016/j.ijfatigue.2018.05.008.

[35] G. Qian, Y. Cao, M. Niffenegger, Y.J. Chao, W. Wu, Comparison of constraint analyses with global and local approaches under uniaxial and biaxial loadings, Eur. J. Mech. A-Solids 69 (2018) 135-146.

[36] G. Qian, W.S. Lei, M. Niffenegger, V.F. Gonzalez, On the temperature independence of statistical model parameters for cleavage fracture in ferritic steels, Philos. Mag. 98 (2018) 959-1004.

[37] G. Lesiuk, P. Kucharski, J.A.F.O. Correia, et al., Mixed mode (I+II) fatigue crack growth in puddle iron, Eng. Fract. Mech. 185 (2017) 175-192.

[38] R. Wang, D. Li, D. Hu, et al., A combined critical distance and highly-stressed-volume model to evaluate the statistical size effect of the stress concentrator on low cycle fatigue of TA19 plate, Int. J. Fatigue 95 (2017) 8-17.

[39] J.F. Wen, T. Tu, F.Z. Xuan, X.W. Zhang, X.L. Gao, Effects of stress level and stress state on creep ductility: mechanism, modelling and test, J. Mater. Sci. Technol. 
32 (2016) 695-794.

[40] S.C. Wu, Z.W. Xu, Y.X. Liu, G.Z. Kang, Z.X. Zhang, On the residual life assessment of high-speed railway axles due to induction hardening, Int. J. Rail Transp. (2018) in press https://doi.org/10.1080/23248378.2018.1427008.

[41] Y. Wang, W. Du, Y. Luo, A mean plastic strain fatigue-creep life prediction and reliability analysis of AISI H13 based on energy method, J. Mater. Res. 32 (22) (2017) 4254-4262.

[42] D. Meng, S. Yang, Y. Zhang, S.P. Zhu, Structural reliability analysis and uncertainties-based collaborative design and optimization of turbine blades using surrogate model, Fatigue Fract. Eng. Mater. Struct. (2018) (in press).

[43] S.P. Zhu, S. Foletti, S. Beretta, Probabilistic framework for multiaxial LCF assessment under material variability, Int. J. Fatigue 103 (2017) $371-385$.

[44] S.P. Zhu, Q. Lei, Q.Y. Wang, Mean stress and ratcheting corrections in fatigue life prediction of metals, Fatigue Fract. Eng. Mater. Struct. 40 (9) (2017) $1343-1354$

[45] A. Ince, A mean stress correction model for tensile and compressive mean stress fatigue loadings, Fatigue Fract. Eng. Mater. Struct. 40 (6) (2017) 939-948.

[46] S.P. Zhu, Q. Lei, H.Z. Huang, Y.J. Yang, W. Peng, Mean stress effect correction in strain energy-based fatigue life prediction of metals, Int. J. Damage Mech. 26 (8) (2017) 1219-1241.

[47] S.P. Zhu, Z.Y. Yu, Q. Liu, A. Ince, Strain energy-based multiaxial fatigue life prediction under normal-shear stress interaction, Int. J. Damage Mech. (2018), https://doi.org/10.1177/1056789518786031 (in press).

[48] S. Xu, S.P. Zhu, Y.Z. Hao, D. Liao, Critical plane-based multiaxial fatigue life prediction of turbine disc alloys by refining normal stress sensitivity, J. Strain Anal. Eng. Des. (2018) in press https://doi.org/10.1177/0309324718779922.

[49] Z.R. Wu, X.T. Hu, Y.D. Song, Multiaxial fatigue life prediction for titanium alloy TC4 under proportional and nonproportional loading, Int. J. Fatigue 59 (2014) $170-175$.

[50] Z.R. Wu, X. Li, L. Fang, Y.D. Song, Evaluation of multiaxial fatigue life prediction criteria for Ni-based superalloy GH4169, Proc. IMechE. Part C 232 (10) (2018) $1823-1837$

[51] Z.Y. Yu, S.P. Zhu, Q. Liu, Y. Liu, A new energy-critical plane damage parameter for multiaxial fatigue life prediction of turbine blades, Materials 10 (5) (2017) 513.

[52] W. Peng, L. Shen, Y. Shen, Q. Sun, Reliability analysis of repairable systems with recurrent misuse-induced failures and normal-operation failures, Reliab. Eng. Syst. Saf. 171 (2018) 87-98.

[53] W. Peng, Y. Li, Y.J. Yang, et al., Bivariate analysis of incomplete degradation observations based on inverse Gaussian processes and copulas, IEEE Trans. Reliab. 65 (2) (2016) 624-639.

[54] H. Xu, W. Li, M. Li, et al., Multidisciplinary robust design optimization based on time-varying sensitivity analysis, J. Mech. Sci. Technol. 32 (3) (2018) 1195-1207. 